\title{
La pédagogie dans les Facultés de médecine françaises
}

\section{$\bigcirc$}

os vies sont faites de hasards successifs que nous réussi ssons parfois à relier. Jeune interne, je participais, un peu par curiosité, à une réunion sur la formation médicale. U ne discussion un peu vive entre deux Q uébécois, m'a profondément marqué : I'un disait à l'autre avec commisération, " toi tu fais de la pédagogie, mais moi, je fais de l'enseignement ! » II y avait là tout le malentendu que les $F$ acultés françaises ont entretenu pendant des siècles et que nos cousins québécois nous ont ai dés à lever. Être pédagogue était consi déré comme un don, à quoi bon l'apprendre? à quoi bon y réfléchir ? à quoi bon mettre en jeu nos habitudes? Les choses ont heureusement changé, plus personne ne discute la nécessité d'une formation à la pédagogie. D e façon corollaire, des nouvel les méthodes sinstallent et les efforts en enseignement pourront être reconnus dans notre systeme français. $M$ ais tout ceci se fait avec plus de lenteur, et de difficultés, que désiré...

\section{Former les enseignants à la pédagogie.}

Cela fait longtemps que l'on parle, en France, de la nécessité d'une formation à la pédagogie pour les médecins, mais c'est depuis seulement une quinzaine d'années, que les initiatives ont rédlement pris corps. 


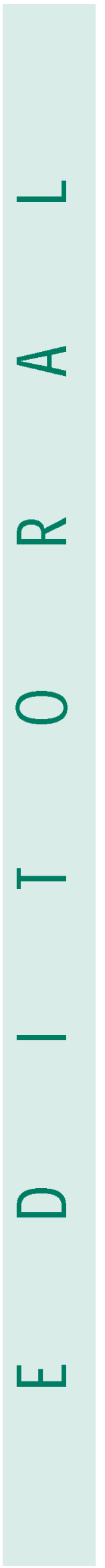

- Comme pour la formation continue, les médecins généralistes ont été un élément moteur de cette mobilisation. II faut leur rendre hommage, en particulier, à Albert H ercek et à G uy Scharf, leurs efforts ont permis de créer l'E cole de Riom, où se sont succédé des générations de généralistes. $N$ ous assistions en fait aux balbutiements de la nouvelle discipline universitaire qu'était la médecine géné rale; les pionniers voulaient $s$ donner les armes que leurs collègues spécialistes avaient souvent dédaigné de prendre.

- Est-ce dire que les généralistes étai ent seuls dans ce combat ? II n'en est rien, d'ailleurs certains universitaires avaient participé de façon active à la création de l'Ecole de Riom, comme Alain Bernadou et Jean-Michel Chabot. Eux-mêmes, depuis des années, multipliai ent les actions dans ce domaine, et avaient inspiré bon nombre de leurs collègues. C'est ainsi que sont apparues, dans de nombreuses Facultés, des initiatives, souvent sous forme de diplômes inter-universitaires (DIU) de pédagogie médicale que ce soit dans l'Est ou dans I'O uest de la France, Certaines disciplines, comme I'anatomie par exemple, ont toujoursmaintenu des exigences pédagogiques pour les nominations, ce qui obligeait leurs candidats à des préparations intensives.

- La Conférence Internationale des doyens en médecine francophones (Ia CID M EF) a, elle aussi, jouéun rôle décisif, grâce à son cercle des experts en pédagogie, dirigé par Jacques Barrier. Le rapport de ce groupe a clarifié les phases de formation des enseignants en trois étapes, la première, celle du formateur débutant (notre chef de clinique), la deuxième, celle del'enseignant confirmé (titulaire), la troi sième étape est celle du pédagogue " expert » personneressource de nosFacultés

- La Conférence des doyens des Facultés françai ses, par l'intermédiaire de sa commission de pédagogie, est en train de fédérer les initiatives locales de formation des jeunes enseignants et souhaite mettre en place des formations nationales pour les plus motivés.

- Lors de la réflexion sur la réforme du deuxième cycle des études françai ses, il y a deux ans, les promoteurs, Gérard Lévy et Bruno Varet avaient prévu de rendre obligatoire la formation pédagogique des enseignants en médecine. $H$ élas, cette mesure, ardemment souhaitée par la Conférence des $D$ oyens de $F$ rance, n'a pas été confirmée.

\section{Rendre la pédagogie vivante dans nos F acultés.}

On sait que la pédagogie est particulièrement complexe en médecine, tant les exigences de l'éducation sont nombreuses. II faut savoir beaucoup de choses, il faut bien utiliser ses connaissances, et en faire des éléments de décision. II faut savoir regarder, palper, écouter, opérer. II faut savoir se comporter. Les réponses pédagogi ques ne peuvent être que multiples. II faut conserver celles qui nous sont utiles, quitte à les améliorer, et introduire toutes celles qui nous manquent.

- II n'est pasquestion de renoncer aux cours magis traux dans nos Facultés. Simplement, ils doivent perdre leur importance écrasante et leur prétention à une impossible exhaustivité. Ils doivent donc se transformer en conférences d'introduction et de conclusion, permettre d'approfondir des notions difficiles, permettre lesinterfaces entre les différents domaines.

- Il n'est pas non plus question d'abandonner le compagnonnage, apprentissage nature, qui permet de rester toujours dans le réd, mais qui mérite d'être mieux codé et mieux évalué

- Il est nécessaire d'installer les méthodes qui donnent aux étudiants une initiative, une autonomie, qui leur permettent d'apprendre à apprendre. L'apprentissage par problèmes, l'apprentissage au raisonnement clinique, font partie de ces méthodes; nous nous réjoui ssons que le Conseiller actuel de notre ministre de l'E ducation en matière de médecine soit le doyen Philippe Lauret qui, le premier en France, avait introduit ces nouvelles pratiques dans son établissement.

- L'évaluation de notre efficience doit se traduire aussi bien dans celle des jeunes médecins que nous formons, que dans celle de nos établi ssements; des efforts importants sont réalisés, ils rejoignent la préoccupation de l'évaluation de notre système de soins, mais l'essentiel reste à faire. $\mathrm{N}$ ous devons donc nous rapprocher de notre agence, I'AN AES, pour potentialiser notre efficacité N ous devons 


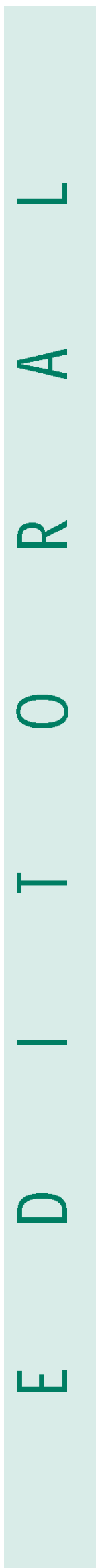

aussi participer aux évaluations des établissements pratiquées par la CID M EF.

- Des départements de pédagogie doivent naître dans nos Facultés. Animés par les enseignants les plus motivés, formés comme personnalités ressources, ces départements doivent être les laboratoires, qui rassemblent les initiatives, facilitent la réflexion et l'évaluation.

\section{Rendre la pédagogie attractive pour les enseignants}

$\mathrm{N}$ ous ne pouvons décréter la pédagogie dans la vie de l'enseignant, il doit être motivé pour se l'approprier. M ais comment faire quand on sait qu'enseigner n'est pas sa vocation première, car la plupart du temps c'est le fait de devenir médecin et non enseignant en médecine, qui a déterminé ses choix. II paraît utile d'incorporer la pédagogie aux diffé rents processus de nomination et de promotion de nos professeurs.

- Recrutement des enseignants : le parcours des candidats en France est loin d'être facile; il doit concilier ses devoi rs hospitaliers, activités de service, gardes, avec une préparation intense aux épreuves qu'il aura à subir. La recherche y joue un rôle déterminant et les exigences des nominations relè vent maintenant plus de l'impact factor que de tout autre paramètre. Le plus haut diplôme de I'université française sappelle d'ailleurs HDR, habilitation à diriger des recherches. En France, chaque candi dat au professorat se soumet à un jury national de sa discipline, la sous-section correspondante de la Commission $\mathrm{N}$ ationale des U niversités (CNU ). Les sous sections font un excellent travail qui permet de jauger le candidat sur le plan de ses recherches, de son aptitude à communiquer sous forme d'exposés; en fait la capacité pédagogique réelle et la formation à la pédagogi e ne sont qu'exceptionnellement estimées. II n'est pas question de surcharger des épreuves qui découragent beaucoup de jeunes, même brillants plus tentés par des situations obtenues plus facilement et bien plus lucratives. II n'est pas question non plus d'abaisser le niveau des recherches. $M$ ais la pédagogie doit être présente, soit dans les recherches elles-mêmes, soit dans le parcours et I'on pourrait envisager que pour certains, I'H D R puisse être remplacée par une

$H D E$, jouant le même rôle dans le domaine de I'enseignement.

- Promotion des enseignants : actuellement la pédagogie ne compte pas. C'est là où l'effort doit donc être le plus grand. Chaque promotion devrait reposer sur la certitude d'une réelle implication dans ce domaine. Organisation de manifestations dans le domaine de la pédagogie, mise en place de systèmes d'éducation dans les services, créations de cydes de formation continue, mise en route de sites Internet d'éducation, participations aux départements de pédagogie des Facultés serviraient ainsi de facteurs favorisant les promotions. $N$ ous attendons beaucoup de discussions avec les sous-sections des CNU sur ces sujets.

Les départements de pédagogie, et leurs « personnes ressources » sont probablement les clés du progrès dans chaque F aculté. A condition de ne pasimiter ce que di sait 0 scar Wilde: « Je vis dans la terreur de ne pas être incompris » donc en évitant des dis cours pédagocratiquesqui fleurissent ici ou là, l'expert en pédagogie doit y favoriser une approche humaniste et même hédoniste de sa di scipline : c'est la seule voie qui lui ralliera enseignants et étudiants. LesF acultés françaises se sont donc engagées sur le chemin de la pédagogie. Beaucoup reste à faire, mais la détermination de la Conférence des D oyens est claire : celle d'avancer au plus vite.

Jacques RO LAN D

Président de la Conférence des $D$ oyens des $F$ acultés de $M$ édecine $F$ rançaises 